\title{
Pre- and Postoperative Capecitabine Without thencertor With Oxaliplatin in Locally Advanced Rectal Cancer: PETACC 6 Trial by EORTC GITCG and ROG, AIO, AGITG, BGDO, and FFCD
}

\begin{abstract}
Hans-Joachim Schmoll, MD, PhD ${ }^{1}$; Alexander Stein, MD²; Eric Van Cutsem, MD, PhD³ Timothy Price, MBBS, DHIthSc ${ }^{4}$;

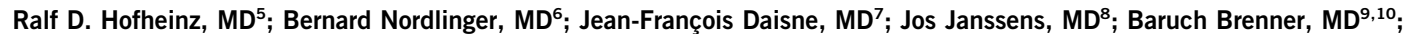
Hans Reinel, MD ${ }^{11}$; Stephan Hollerbach, MD ${ }^{12}$; Karel Caca, MD, PhD ${ }^{13}$; Florian Fauth, MD ${ }^{14}$; Carla V. Hannig, MD ${ }^{15}$; John Zalcberg, MBBS, PhD ${ }^{16}$; Niall Tebbutt, BM, BCh, PhD ${ }^{17}$; Murielle E. Mauer, PhD ${ }^{18}$; Sandrine Marreaud, MD ${ }^{18}$; Manfred P. Lutz, MD ${ }^{19}$; and Karin Haustermans, MD $^{3}$
\end{abstract}

PURPOSE The PETACC 6 trial investigates whether the addition of oxaliplatin to preoperative capecitabine-based chemoradiation and postoperative capecitabine improves disease-free survival (DFS) in locally advanced rectal cancer.

METHODS Between November 2008 and September 2011, patients with rectal adenocarcinoma within $12 \mathrm{~cm}$ from the anal verge, T3/4 and/or node positive, were randomly assigned to 5 weeks preoperative capecitabinebased chemoradiation (45-50.4 Gy) followed by six cycles of adjuvant capecitabine, both without (control arm, 1) or with (experimental arm, 2) oxaliplatin. The primary end point was improvement of 3-year DFS by oxaliplatin from $65 \%$ to $72 \%$ (hazard ratio [HR], 0.763).

RESULTS A total of 1,094 patients were randomly assigned (intention to treat), and 1,068 eligible patients started their allocated treatment (arm 1, 543; arm 2, 525), with completion of protocol treatment in 68\% (arm 1) $v$ $54 \%$ (arm 2). A higher rate of grade $3 / 4$ adverse events was reported in the experimental arm ( $14.4 \%$ v $37.3 \%$ and $23.4 \% v 46.6 \%$ for neoadjuvant and adjuvant treatment, respectively). At a median follow-up of 68 months (interquartile range, 58-74 months), 157 and 156 DFS events were observed in arms 1 and 2, respectively (adjusted HR, 1.02; $95 \% \mathrm{Cl}, 0.82$ to $1.28 ; P=.835$ ). Three-year DFS rate was not different, with $76.5 \%(95 \% \mathrm{Cl}, 72.7 \%$ to $79.9 \%)$ in arm 1 , which is higher than anticipated, and $75.8 \%(95 \% \mathrm{Cl}, 71.9 \%$ to $79.3 \%$ ) in arm 2. The 7-year DFS and overall survival (OS) rates were not different as well, with DFS of $66.1 \% \mathrm{v}$ $65.5 \%(H R, 1.02)$ and OS of $73.5 \% \vee 73.7 \%(H R, 1.19)$ in arms 1 and 2 , respectively. Subgroup analyses revealed heterogeneity in treatment effect according to German versus non-German site location, without detectable confounding factors in multivariable analysis.

CONCLUSION The addition of oxaliplatin to preoperative capecitabine-based chemoradiation and postoperative adjuvant chemotherapy impairs tolerability and feasibility and does not improve efficacy.

ASSOCIATED

CONTENT

Appendix

Data Supplement

Protocol

Author affiliations

and support

information (if

applicable) appear

at the end of this

article.

Accepted on August 25,2020 and

published at

ascopubs.org/journal/ jco on October 1 ,

2020: DOI https://doi. org/10.1200/JCO.20. 01740
J Clin Oncol 38. $\odot 2020$ by American Society of Clinical Oncology

\section{INTRODUCTION}

In localized rectal cancer stage II/III (cT3/4 or any lymph node involvement), neoadjuvant chemoradiation therapy (CRT) with fluoropyrimidine, followed by total mesorectal excision (TME) and adjuvant fluoropyrimidine-based chemotherapy, has become a standard of care. ${ }^{1-3}$ To improve local control and distant failure, the PETACC 6 trial, alongside several other trials, was launched to assess the impact of the addition of oxaliplatin to neoadjuvant CRT and in parallel with CAO/ARO/AIO-04 to adjuvant chemotherapy. ${ }^{4-7}$ PETACC 6 was initiated in 2008 to compare neoadjuvant CRT with concurrent capecitabine, followed by TME and adjuvant capecitabine, with neoadjuvant CRT with capecitabine and oxaliplatin (CAPOX), followed by TME and adjuvant CAPOX. The chemotherapy chosen was based on prior data showing the feasibility of CRT with capecitabine with or without oxaliplatin and the proven similar efficacy of fluorouracil (5FU) and capecitabine as single agent in adjuvant colon cancer. ${ }^{8-15}$

\section{METHODS}

\section{Patients}

Eligible patients were age $\geq 18$ years with pathologically confirmed rectal cancer, inferior margin up to 


\section{CONTEXT}

\section{Key Objective}

To define the impact of adding oxaliplatin to perioperative capecitabine-based neoadjuvant chemoradiation therapy and adjuvant chemotherapy in locally advanced rectal cancer.

\section{Knowledge Generated}

The addition of oxaliplatin to perioperative multimodality treatment impairs feasibility and tolerability and does not improve short-term (pathologic response) or long-term outcome (survival).

\section{Relevance}

There is no role for oxaliplatin in neoadjuvant capecitabine-based chemoradiation therapy and postoperative adjuvant chemotherapy.

$12 \mathrm{~cm}$ from the anal verge (by rigid proctoscopy), and clinical T3/4 or any $T$ with lymph node involvement ( $\mathrm{cN}+$; by endorectal ultrasound [EUS] and/or pelvic magnetic resonance imaging [MRI] or, if MRI not available, computed tomography [CT] plus EUS). Pretreatment CT scan of the chest and abdomen was required to exclude metastatic disease. Additional inclusion criteria were Eastern Cooperative Oncology Group performance status (ECOG PS) 0-2 and adequate hematologic, liver, and renal function. Exclusion criteria were metastatic disease, prior pelvic radiotherapy (RT) or chemotherapy, other cancers within the past 5 years, pregnancy, lactation, clinically significant cardiac disease, and known peripheral neuropathy.

\section{Random Assignment}

Patients were centrally (European Organisation for Research and Treatment of Cancer [EORTC] data center) randomly assigned using a 1:1 allocation ratio to the control arm with pre- and postoperative capecitabine or to the experimental arm with pre- and postoperative CAPOX. Random assignment was not blinded and stratified for institution, clinical T category (T1-3 $v$ T4), clinical nodal status (NX $v$ NO $v$ N1-2), distance from the tumor to the anal verge ( $\leq 5 \mathrm{~cm} v>5 \mathrm{~cm}$ ), and locoregional staging method (EUS + MRI $v$ EUS + CT $v$ MRI alone).

\section{Procedure}

In the control arm, neoadjuvant CRT with 45 or 50.4 Gy with concurrent capecitabine $2 \times 825 \mathrm{mg} / \mathrm{m}^{2}$ twice daily without weekends, followed by TME and adjuvant capecitabine with $1,000 \mathrm{mg} / \mathrm{m}^{2}$ twice daily on days $1-15$ every 3 weeks for six cycles was applied. In the experimental (CAPOX) arm, oxaliplatin was added to the same capecitabine dose during neoadjuvant CRT with $50 \mathrm{mg} / \mathrm{m}^{2}$ for days $1,8,15,22$, and 29 and during adjuvant chemotherapy with $130 \mathrm{mg} / \mathrm{m}^{2}$ on day 1 every 3 weeks for six cycles (Data Supplement, online only).

RT consisted of 45 Gy in 25 fractions ( 1.8 Gy daily, MondayFriday), delivered with a minimum energy of 6-MV photons through a three-field or four-field box technique to the primary tumor and to mesorectal, presacral, and internal iliac lymph nodes. An additional dose of 5.4 Gy could be given using the same fields or as a boost to the macroscopic tumor (primary and nodes) up to a total dose of 50.4 Gy in 28 fractions (1.8 Gy per fraction). Centers had to choose one option ( 45 or $50.4 \mathrm{~Gy}$ ) and adopt it for both arms for the entire study. Surgery was planned 4-6 weeks after completion of neoadjuvant treatment. Before surgery, a clinical evaluation of response was performed using digital rectal examination; pelvic MRI; or, if MRI was not available, CT, EUS, and abdominal CT. Surgical technique was left to the surgeon, and TME was regarded as standard of care. Pathology was based on TNM classification International Union Against Cancer (UICC) sixth edition, including number of examined and involved lymph nodes and status of proximal, distal, and circumferential resection margins.

Adjuvant treatment of 4.5 months with capecitabine with or without oxaliplatin was started within 6-8 weeks postoperatively. Follow-up measures were carcinoembryonic antigen (every 3 months for years 1-3, every 6 months for years $4-5$, chest CT every 12 months and abdominal CT or ultrasound every 6 months for years 1-3; for years 4-5, only EUS every 12 months). In case of no complete colonoscopy preoperatively, colonoscopy was performed 6 months and 3 years after adjuvant treatment.

Patients were monitored weekly during CRT and before each adjuvant treatment cycle for adverse events, vital signs, ECOG PS, and laboratory measurements. Dose modifications were done according to toxicity (National Cancer Institute Common Terminology Criteria for Adverse Events [version 3.0; CTCAE v3.0]). Patients were followed for overall survival (OS) for at least 5 years after the end of postoperative study treatment or death.

\section{Statistical Analysis}

The primary end point of disease-free survival (DFS) was defined from random assignment to the first event: locoregional failure, metastatic recurrence, secondary colorectal cancer, or death. R2 resection distant metastases at surgery were considered treatment failures. 
The trial was planned to detect a 3-year DFS improvement from $65 \%$ to $72 \%$ (hazard ratio [HR], 0.763), with $80 \%$ power at a two-sided $5 \%$ significance level on the basis of an interim analysis for early efficacy at year 3 after the start of the recruitment or at 200 events. Accounting for that interim analysis, the final analysis was to be conducted with a one-sided $\alpha$ of 0.023 on the basis of 440 events.
Assuming 2.8 years of recruitment and 3 additional years of follow-up, a total of 1,090 patients were to be randomly assigned to observe the required 440 events.

Secondary end points were OS, locoregional or distant failure, pathologic downstaging (ypTO-2NO) rate, pathologic complete remission (pCR; ypTONO) rate, tumor regression grade, histopathologic RO resection rate, sphincter

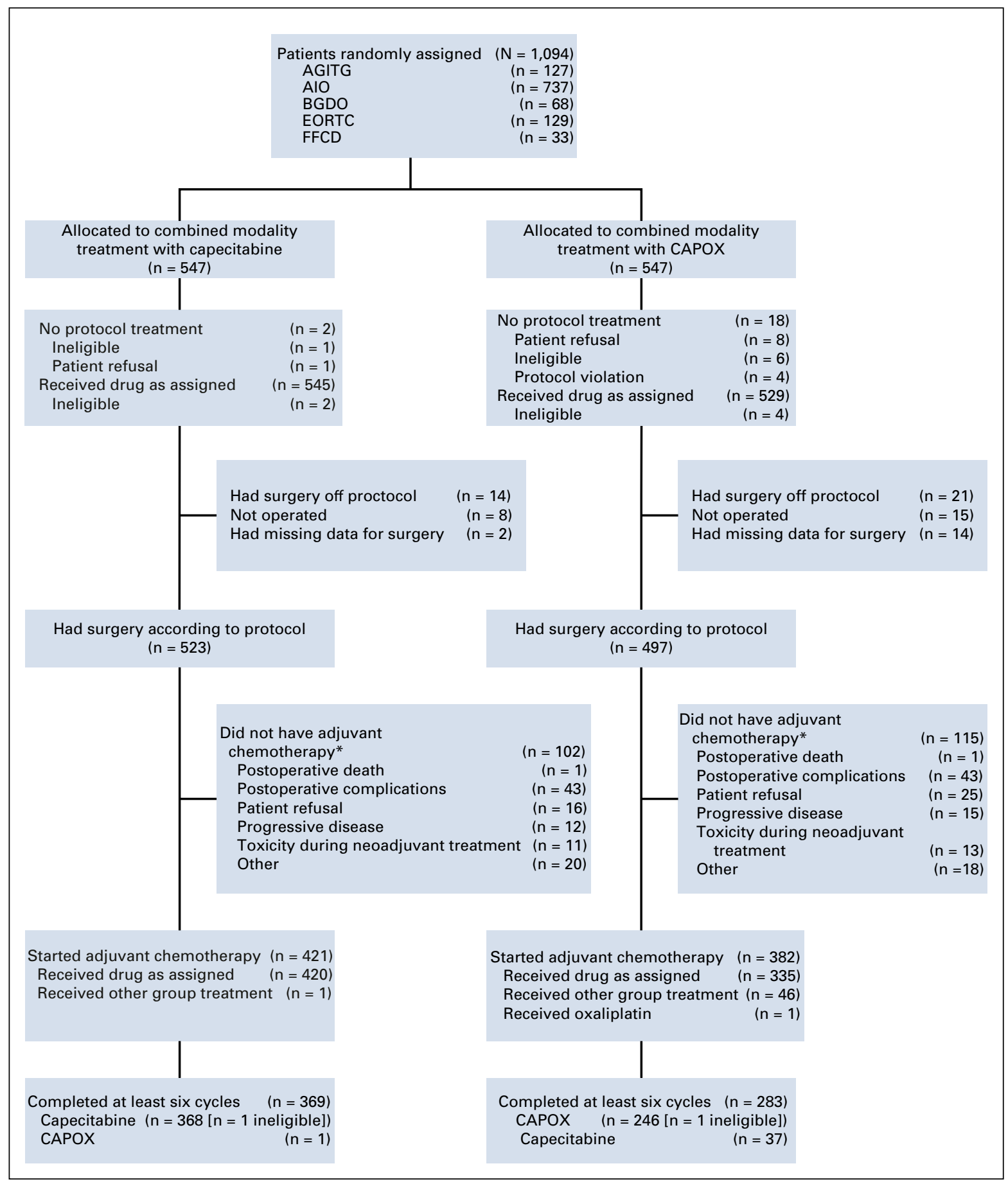

FIG 1. CONSORT diagram. AGITG, Australasian Gastro-Intestinal Trials Group; AIO, Arbeitsgemeinschaft Internistische Onkologie; BGDO, Belgian Group of Digestive Oncology; CAPOX, capecitabine and oxaliplatin; EORTC, European Organisation for the Research and Treatment of Cancer; FFCD, Fédération Francophone de Cancérologie Digestive. 
TABLE 1. Baseline Characteristics

Characteristic

Cape + RT, No. (\%)

CAPOX + RT, No. (\%)

\begin{tabular}{|c|c|c|}
\hline No. of patients & 547 & 547 \\
\hline Median age, years (range) & $62(26-87)$ & $62(23-82)$ \\
\hline \multicolumn{3}{|l|}{ Sex } \\
\hline Male & $394(72.0)$ & $380(69.5)$ \\
\hline Female & $153(28.0)$ & $167(30.5)$ \\
\hline \multicolumn{3}{|l|}{ ECOG PS } \\
\hline 0 & $420(76.8)$ & $432(79.0)$ \\
\hline 1 & $126(23.0)$ & $108(19.7)$ \\
\hline 2 & $1(0.2)$ & $7(1.3)$ \\
\hline \multicolumn{3}{|l|}{ cT } \\
\hline cT1 & $3(0.5)$ & $2(0.4)$ \\
\hline cT2 & $36(6.6)$ & $33(6.0)$ \\
\hline cT3 & $466(85.2)$ & $469(85.7)$ \\
\hline cT4 & $42(7.7)$ & $43(7.9)$ \\
\hline \multicolumn{3}{|l|}{$\mathrm{cN}$} \\
\hline $\mathrm{cNO}$ & $118(21.6)$ & $120(21.9)$ \\
\hline $\mathrm{cN} 1$ & $295(53.9)$ & $296(54.1)$ \\
\hline cN2 & 98 (17.9) & $93(17.0)$ \\
\hline cNX & $36(6.6)$ & $38(6.9)$ \\
\hline \multicolumn{3}{|l|}{ TNM stage } \\
\hline II & $116(21.2)$ & $120(21.9)$ \\
\hline III & $392(71.7)$ & $386(70.6)$ \\
\hline cT3-4, cNX & $35(6.4)$ & $38(6.9)$ \\
\hline Missing & $4(0.7)$ & $3(0.5)$ \\
\hline \multicolumn{3}{|c|}{ Distance of tumor to anal verge, $\mathrm{cm}$} \\
\hline$\leq 5$ & $236(43.1)$ & $237(43.3)$ \\
\hline$>5$ & $311(56.9)$ & $310(56.7)$ \\
\hline \multicolumn{3}{|l|}{ MRI available at the center } \\
\hline No & $66(12.1)$ & $56(10.2)$ \\
\hline Yes & 481 (87.9) & 491 (89.8) \\
\hline \multicolumn{3}{|c|}{ Locoregional staging performed by } \\
\hline $\mathrm{EUS}+\mathrm{MRI}$ & $229(41.9)$ & $224(41.0)$ \\
\hline EUS + CT scan & $192(35.1)$ & 207 (37.8) \\
\hline MRI alone & $126(23.0)$ & $116(21.2)$ \\
\hline
\end{tabular}

Abbreviations: Cape, capecitabine; CAPOX, capecitabine and oxaliplatin; CT, computed tomography; ECOG PS, Eastern Cooperative Oncology Group performance status; EUS, endorectal ultrasound; MRI, magnetic resonance imaging; RT, radiotherapy.

preservation rate, perioperative complication rate, and toxicity according to CTCAE v3.0 (refer to the Appendix, online only, for specifications of statistical analyses).

Because of the higher toxicity in the experimental arm, the safety data were reviewed (unplanned review) by the EORTC independent data monitoring committee (IDMC) in May 2011, which recommended continuation of the trial given that the targeted DFS benefit was judged to still represent a positive risk:benefit ratio. However, the events had not accumulated at the pace expected, given that the 3-year DFS was higher in the control arm than anticipated. The interim analysis was performed in May 2013 at 225 events. The EORTC IDMC recommended the early release of the data on basis of futility while continuing follow-up until the planned final analysis.

\section{RESULTS}

From November 2008 to September 2011, 1,094 patients ( $n=547$ in each arm) were randomly assigned in 181 centers in Australia, Belgium, France, Germany, Israel, and 
TABLE 2. Treatment Exposure During Neoadjuvant CRT and Adjuvant Chemotherapy

\begin{tabular}{|c|c|c|c|c|c|c|}
\hline \multirow[b]{2}{*}{ Treatment } & & \multicolumn{2}{|c|}{ 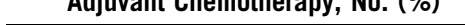 } \\
\hline & \multicolumn{2}{|c|}{$\begin{array}{c}\text { Capecitabine + RT } \\
\left(\mathrm{n}=545^{\mathrm{a}}\right)\end{array}$} & \multicolumn{2}{|c|}{$\begin{array}{l}\text { CAPOX + RT } \\
\left(n=532^{a}\right)\end{array}$} & $\begin{array}{c}\text { Capecitabine }+ \\
\text { RT } \\
(n=420)\end{array}$ & $\begin{array}{l}\text { CAPOX + RT } \\
(n=335)\end{array}$ \\
\hline \multicolumn{7}{|l|}{ Planned RT, Gy } \\
\hline 45 & \multicolumn{2}{|c|}{$109(20.0)$} & \multicolumn{2}{|c|}{$113(21.2)$} & & \\
\hline 50.4 (same fields) & \multicolumn{2}{|c|}{$156(28.6)$} & \multicolumn{2}{|c|}{$141(26.5)$} & & \\
\hline 50.4 (boost) & \multicolumn{2}{|c|}{$276(50.6)$} & \multicolumn{2}{|c|}{$275(51.7)$} & & \\
\hline 50.4 (unknown) & \multicolumn{2}{|c|}{$4(0.7)$} & \multicolumn{2}{|c|}{$3(0.6)$} & & \\
\hline Received planned dose & \multicolumn{2}{|c|}{$534(98.0)$} & \multicolumn{2}{|c|}{$499(93.8)$} & & \\
\hline RT not started & \multicolumn{2}{|c|}{$0(0.0)$} & \multicolumn{2}{|c|}{$1(0.2)^{b}$} & & \\
\hline \multicolumn{7}{|l|}{ RT prematurely stopped } \\
\hline Toxicity & \multicolumn{2}{|c|}{$8(1.5)$} & \multicolumn{2}{|c|}{19 (3.6) } & & \\
\hline \multicolumn{7}{|l|}{ Temporary interruption } \\
\hline Toxicity & \multicolumn{2}{|c|}{$22(4.0)$} & \multicolumn{2}{|c|}{$67(12.6)$} & & \\
\hline \multicolumn{5}{|l|}{ Capecitabine } & \multirow[t]{2}{*}{$421 \mathrm{c}$} & $381 \mathrm{c}$ \\
\hline Relative dose intensity (\%) & $\begin{array}{l}45 \text { Gy } \\
(n=222)\end{array}$ & $\begin{array}{l}50.4 \text { Gy } \\
(n=855)\end{array}$ & $\begin{array}{l}45 \text { Gy } \\
(n=222)\end{array}$ & $\begin{array}{l}50.4 \text { Gy } \\
(n=855)\end{array}$ & & \\
\hline Median & 92.1 & 97.8 & 90.8 & 95.1 & 94.1 & 89.1 \\
\hline Range & $52.8-115.3$ & $53.1-140.0$ & $26.7-28.6$ & 28.4-145.8 & $30.5-124.5$ & $10.5-112.3$ \\
\hline $\begin{array}{l}\text { Any toxicity-related dose } \\
\text { reduction or withdrawal }\end{array}$ & \multicolumn{2}{|c|}{$57(10.5)$} & \multicolumn{2}{|c|}{165 (31.0) } & $101(24.0)$ & 142 (37.3) \\
\hline Non-hematological toxicity & \multicolumn{2}{|c|}{$52(9.5)$} & \multicolumn{2}{|c|}{$151(28.4)$} & & \\
\hline Oxaliplatin, No. of patients & & & & & & $336^{d}$ \\
\hline $\begin{array}{l}\text { Median relative dose, } \\
\% \text { (range) }\end{array}$ & \multicolumn{4}{|c|}{$98.7(0.0-128.0)$} & & $84.4(11.4-300.9)$ \\
\hline $\begin{array}{l}\text { Any toxicity-related dose } \\
\text { reduction or withdrawal }\end{array}$ & \multicolumn{4}{|c|}{$119(22.4)$} & & 161 (47.9) \\
\hline
\end{tabular}

Abbreviations: CAPOX, capecitabine and oxaliplatin; CRT, chemoradiation therapy; RT, radiotherapy.

aPatients who started preoperative treatment.

${ }^{\mathrm{b}}$ Discontinuation because of a serious adverse event.

${ }^{c}$ All patients operated on within protocol who started postoperative capecitabine.

${ }^{\mathrm{d}}$ All patients operated on within protocol who started postoperative oxaliplatin.

New Zealand. A total of 1,068 patients were eligible (543 in the control arm and 525 in the experimental arm; Fig 1), with 12 patients ineligible, one unverifiable, four having protocol violations, and nine withdrawing consent. Baseline characteristics were well balanced between arms (Table 1). The local staging at baseline was EUS and MRI in 453 patients (41.4\%), EUS and pelvic CT scan in 399 (36.5\%), and MRI alone in $242(22.1 \%)$.

\section{Neoadjuvant CRT}

RT dose (45 v 50.4 Gy) was balanced between the treatment arms, with $20 \%$ of patients receiving 45 Gy. Completion rates of planned RT were $98 \%$ and $94 \%$ (Table 2). Treatment compliance during neoadjuvant CRT was as expected and well balanced, with the exception of dose modifications for capecitabine $(31 \%$ in the experimental arm $v 10.5 \%$ in the control arm) mainly because of nonhematologic toxicity (Table 2). Accordingly, dose modifications for oxaliplatin in the experimental arm (22.4\%) were correlated to capecitabine modifications $(P<.0001)$. Of note, surgical and pathologic outcomes were not influenced by dose modifications (Appendix Table A1, online only). Furthermore, the rate of grade $3 / 4$ diarrhea was approximately tripled by the addition of oxaliplatin to capecitabine-based RT (Table 3).

\section{Surgery}

Type of surgery as well as postoperative morbidity and mortality did not relevantly differ between arms, with TME in $89 \%$ of both, any postoperative complications $(41.9 \% \mathrm{~V}$ 
TABLE 3. Toxicity During Neoadjuvant CRT and Postoperative Chemotherapy

Neoadjuvant CRT, No. (\%)

\begin{tabular}{|c|c|c|c|c|}
\hline \multirow[b]{2}{*}{ Toxicity (NCI CTCAE v3.0) } & & \\
\hline & Capecitabine + RT & CAPOX + RT & Capecitabine & CAPOX \\
\hline No. of patients & $545^{a}$ & $529^{a}$ & $420^{b}$ & $335^{\mathrm{b}}$ \\
\hline All grade $3-4$ & 79 (14.5) & $197(37.2)$ & 99 (23.6) & $155(46.3)$ \\
\hline Grade 3-4 Gl & $43(7.9)$ & $115(21.7)$ & $32(7.6)$ & $76(22.6)$ \\
\hline Diarrhea & $33(6.1)$ & 98 (18.5) & $23(5.4)$ & $42(12.5)$ \\
\hline Nausea & $3(0.6)$ & $13(2.5)$ & $2(0.5)$ & $17(5.1)$ \\
\hline Vomiting & $3(0.6)$ & $9(1.7)$ & $1(0.2)$ & $18(5.4)$ \\
\hline Stomatitis & $1(0.2)$ & $3(0.6)$ & $2(0.5)$ & $2(0.6)$ \\
\hline Proctitis & $7(1.3)$ & $9(1.7)$ & NA & NA \\
\hline \multicolumn{5}{|l|}{ Grade 3-4 general } \\
\hline Infection & $7(1.3)$ & $14(2.7)$ & $9(2.3)$ & $14(4.2)$ \\
\hline Fatigue & $2(0.4)$ & $20(3.8)$ & $6(1.4)$ & $16(4.8)$ \\
\hline Grade 3-4 renal/genitourinary & $1(0.2)$ & $10(1.9)$ & $10(2.4)$ & $6(1.8)$ \\
\hline Grade 3-4 radiation dermatitis & $8(1.5)$ & $9(1.7)$ & NA & NA \\
\hline Grade 3-4 hand-foot syndrome & $0(0)$ & $1(0.2)$ & $22(5.2)$ & $7(2.1)$ \\
\hline Grade 2-4 neuropathy & $1(0.2)$ & $48(9.1)$ & $12(2.9)$ & $113(33.7)$ \\
\hline
\end{tabular}

Abbreviations: CAPOX, capecitabine and oxaliplatin; CRT, chemoradiation therapy; NA, not applicable; NCI CTCAE v3.0, National Cancer Institute Common Terminology Criteria for Adverse Events (version 3.0); RT, radiotherapy.

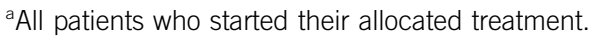

${ }^{b}$ All patients operated on within protocol and who started the allocated postoperative treatment.

$45.9 \%$ in the control $v$ experimental arms, respectively), and mortality in three patients per arm (Table 4). In the intention-to-treat population with data available, the composite end point perioperative complication rate (defined as prolongation of hospitalization $>20$ days after surgery) or a severe event within 30 days of surgery (new hospitalization, re-operation, or death as a result of severe surgeryrelated complication; organ failure; severe bleeding; thromboembolism; major impaired wound healing delaying start of adjuvant chemotherapy by $>8$ weeks; or severe pre- or postoperative treatment-related toxicity inducing a $>8$-week delay of surgery or treatment discontinuation or death) was significantly lower in the control arm (30.6\%) than in the experimental arm (40.3\%; $P=.0009$; Table 4).

\section{Adjuvant Chemotherapy}

In the control arm, $77 \%(n=420)$ started adjuvant capecitabine (with one patient receiving CAPOX) compared to only $61 \%(n=335)$ in the experimental arm starting on adjuvant CAPOX as planned and further $8 \%(n=46)$ on capecitabine only and one patient on oxaliplatin only. Furthermore, of those who started adjuvant chemotherapy, significantly fewer patients in the experimental arm completed the planned duration of 4.5 months ( $74 \% \vee 88 \%$; $P<.0001$ ). Post hoc analysis showed better outcomes in patients starting adjuvant chemotherapy compared with patients without adjuvant chemotherapy after surgery (Appendix Table A2, online only). Toxicity was as expected, with a higher rate of $\mathrm{Gl}$ adverse events and neuropathy in the experimental arm compared with capecitabine alone (Table 3).

\section{Primary and Secondary End Points}

The rates of pathologic tumor downstaging were comparable in both arms (Table 4) (ypTO-2 NO, 44.9\% v 44.4\%; pCR [ypTO NO], $11.6 \%$ v 14.0\%; tumor regression grade [Dvorak 2-4], 70.9\% $\vee 75.8 \%$; and sphincter preservation rate, $71.3 \% \vee 69.9 \%$ in the control $v$ experimental arms, respectively). The pathologic RO resection rate with 93.4\% (control) v 95.8\% (experimental) was not different $(P=.084 ;$ Table 4$)$.

The cumulative incidence of locoregional and distant failure at 5 years were similar in both arms. The 5 -year locoregional failure rate was $8.68 \%(95 \% \mathrm{Cl}, 6.22 \%$ to $11.14 \%)$ in the control arm $v 6.02 \%(95 \% \mathrm{Cl}, 3.93 \%$ to $8.11 \%)$ in the experimental arm ( $P=.204$; Fig $2 A)$. The 5year distant failure rate was $21.43 \%(95 \% \mathrm{Cl}, 17.88 \%$ to $24.97 \%)$ in the control arm $v 19.22 \%(95 \% \mathrm{Cl}, 15.77 \%$ to $22.67 \%)$ in the experimental arm ( $P=.246$; Fig 2B).

Overall, 157 and 156 DFS events were observed in the control and experimental arms, respectively. The 3-year DFS rate was similar with $76.5 \%$ (95\% Cl, $72.7 \%$ to $79.9 \%)$ in the control arm and, thus, higher than the anticipated $65 \%, v 75.8 \%(95 \% \mathrm{Cl}, 71.9 \%$ to $79.3 \%)$ in the experimental arm. The addition of oxaliplatin to the perioperative treatment did not improve DFS (HR, 1.02; $95 \% \mathrm{Cl}, 0.82$ to 1.28; $P=.835$; Fig $2 \mathrm{C}$ ). The 5 - and 7-year DFS rates were 
TABLE 4. Surgical Procedures and Outcome of All Patients Operated on Within the Protocol and Surgical or Pathologic End Points Pertaining to the Surgical Outcome According to Treatment Arm (ITT)

Percentage, ${ }^{a}$ No. (\%)

\begin{tabular}{|c|c|c|c|c|}
\hline & & & & \\
\hline Variable & Cape + RT & CAPOX + RT & $\mathrm{OR}^{\mathrm{b}}(95 \% \mathrm{CI})$ & $P^{\mathrm{b}}$ \\
\hline $\begin{array}{l}\text { Surgical procedures and outcome, No. of } \\
\text { patients operated on within the protocol }\end{array}$ & 523 & 497 & & \\
\hline Type of surgery & & & & \\
\hline Laparoscopy & $153(29.3)$ & $138(27.8)$ & & \\
\hline Laparotomy & $367(70.2)$ & $354(71.2)$ & & \\
\hline Unknown & $3(0.6)$ & $5(1.0)$ & & \\
\hline Surgical procedure & & & & \\
\hline Low anterior resection & $383(73.2)$ & $352(70.8)$ & & \\
\hline Abdominoperineal resection & $136(26.0)$ & $136(27.4)$ & & \\
\hline Other & $4(0.8)$ & $4(0.8)$ & & \\
\hline Missing & $0(0.0)$ & $5(1.0)$ & & \\
\hline Total mesorectal excision & $466(89.1)$ & $445(89.5)$ & & \\
\hline Postoperative morbidity & & & & \\
\hline Any complication ${ }^{b}$ (any grade) & $219(41.9)$ & $228(45.9)$ & & \\
\hline Anastomotic dehiscence & $35(6.7)$ & $35(7.0)$ & & \\
\hline Impaired wound healing & & & & \\
\hline Minor & $49(9.4)$ & $48(9.7)$ & & \\
\hline Major & $38(7.3)$ & $37(7.4)$ & & \\
\hline Bowel obstruction & $15(2.9)$ & $13(2.6)$ & & \\
\hline Fistula & $16(3.1)$ & $13(2.6)$ & & \\
\hline Severe sepsis & $6(1.1)$ & $10(2.0)$ & & \\
\hline Renal failure requiring dialysis & $2(0.4)$ & $6(1.2)$ & & \\
\hline Second surgery for complications & $55(10.5)$ & $54(10.9)$ & & \\
\hline Death due to PETACC 6 surgery & $3(0.6)$ & $3(0.6)$ & & \\
\hline SAE during/as a result of surgery & $102(19.5)$ & $126(25.4)$ & & \\
\hline $\begin{array}{l}\text { End points pertaining to the surgical outcome } \\
\text { according to treatment arm (ITT), No. of patients }\end{array}$ & 547 & 547 & & \\
\hline Perioperative complication rate, \% & 30.6 & 40.3 & 1.53 (1.19 to 1.97$)$ & .0009 \\
\hline Pathologic downstaging (ypT0-2N0) rate, \% & 44.9 & 44.4 & 0.98 (0.77 to 1.25$)$ & .890 \\
\hline pCR (ypTONO) rate, \% & 11.6 & 14.0 & 1.25 (0.87 to 1.79$)$ & .225 \\
\hline Histopathologic R0 resection rate, \% & 95.8 & 93.4 & 0.62 (0.36 to 1.07$)$ & .084 \\
\hline Tumor regression grade (Dworak) & & & 1.28 (0.97 to 1.69$)$ & .077 \\
\hline No/minimal regression $(0,1)$ & 29.1 & 24.2 & & \\
\hline Moderate/good/total regression (2-4) & 70.9 & 75.8 & & \\
\hline Sphincter preservation rate & 71.3 & 69.9 & 0.96 (0.71 to 1.29$)$ & .781 \\
\hline
\end{tabular}

Abbreviations: Cape, capecitabine; CAPOX, capecitabine and oxaliplatin; ITT, intention to treat; OR, odds ratio; pCR, pathologic complete remission; RT, radiotherapy; SAE, serious adverse event.

aPercentages are for patients with available data. Patients not operated on or not receiving a resection were considered to have experienced treatment failure in the analyses.

${ }^{\mathrm{b}} \mathrm{ORs}, 95 \% \mathrm{Cls}$, and $P$ values were computed from a logistic regression model with adjustment for all stratification factors except center. Patients with missing data were removed from the analyses. 


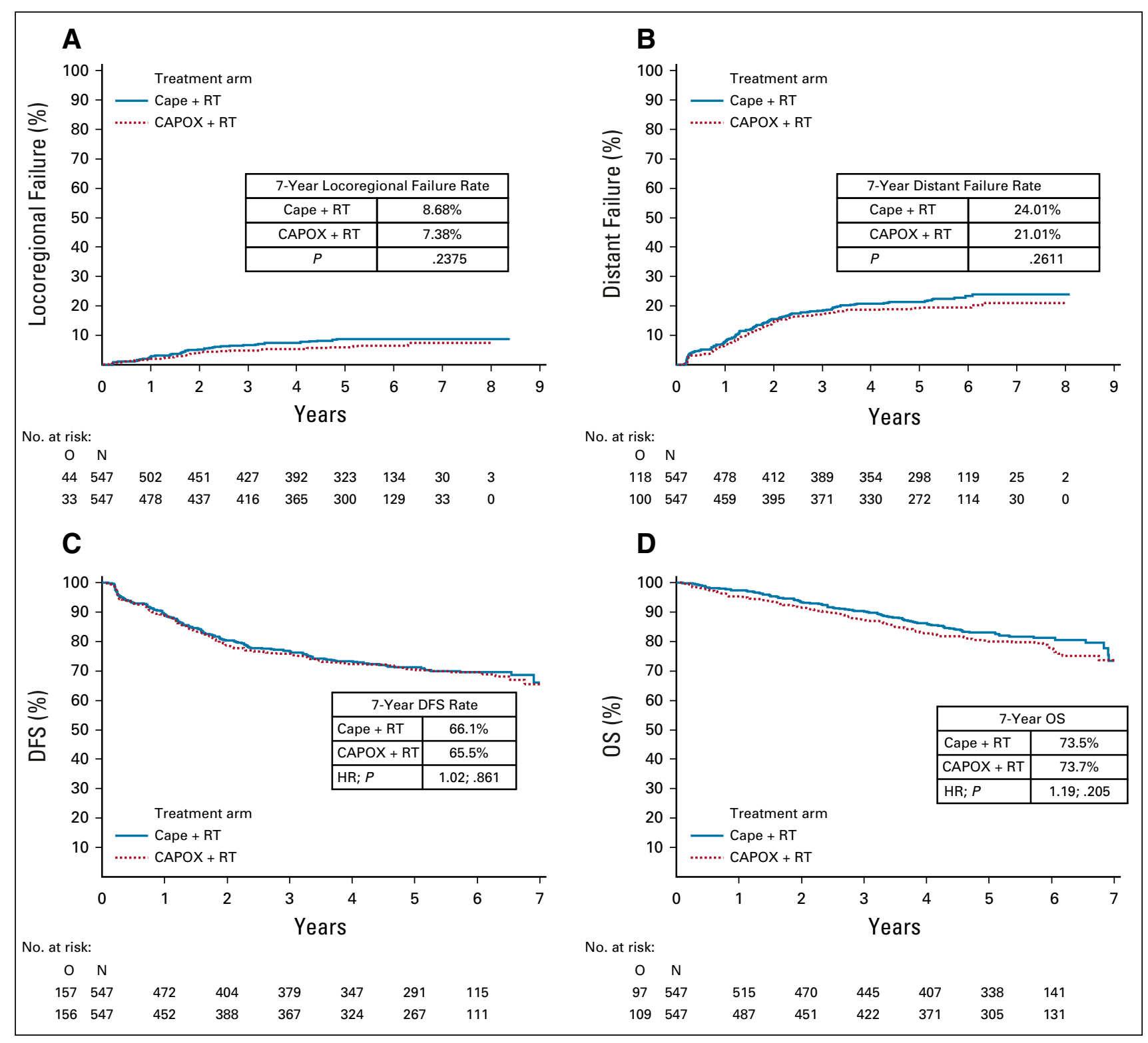

FIG 2. (A) Locoregional and (B) distant failures and (C) disease-free survival (DFS) and (D) overall survival (OS) at 7 years follow-up by treatment arm. Cape, capecitabine; CAPOX, capecitabine and oxaliplatin; HR, hazard ratio; N, number of patients at risk; O, observed events; RT, radiotherapy.

$71.3 \% \vee 70.5 \%$ and $66.1 \% \vee 65.5 \%$ for the control versus experimental arms, respectively. There was no difference in 5-year OS rate $(83.1 \% \vee 80.1 \%)$ and 7 -year OS rate $(73.5 \% \vee 73.7 \%)$ in the control versus experimental arms, respectively $(\mathrm{HR}, 1.19 ; 95 \% \mathrm{Cl}, 0.91$ to $1.57 ; P=.205$; Figs $2 \mathrm{C}$ and $2 \mathrm{D}$ ).

\section{Post Hoc Subgroup Analyses}

Retrospective subgroup analyses did not reveal any heterogeneity in treatment effect on DFS according to major baseline factors, except for country of patient inclusion $(P=.01$; Fig 3$)$. However, multiple tests were performed without adjusting the level of significance.

No impact of the addition of oxaliplatin was noted in stage II (21\% of patients) for DFS (HR, 0.95; $P=.82$ ) or OS (HR, 0.95;
$P=.84)$ and in stage III (72\%; 7\% cT3-4, cNX) for DFS (HR, 1.04; $P=.78$ ) or OS (HR, 1.21, $P=.27$; Data Supplement).

However, strong and inverse differences were observed in patients included at German sites ( $n=737$ ): oxaliplatin with a numerically decreased DFS rate at 5 years $(73.4 \% \mathrm{~V}$ $67.8 \% ; \mathrm{HR}, 1.27 ; P=.092)$ in contrast to non-German sites ( $n=357$ ) with a numerically increased DFS rate at 5 years $(67.0 \%$ v 75.7\%; HR, 0.70; $P=.061$; Data Supplement). Main differences were stage distribution and staging with MRI at baseline (Appendix Table A3, online only) and rates of application of adjuvant chemotherapy (Appendix Table A2).

The multivariable prognostic analysis in pooled treatment arms identified older age ( $>60$ years), cT3-4 disease, and 


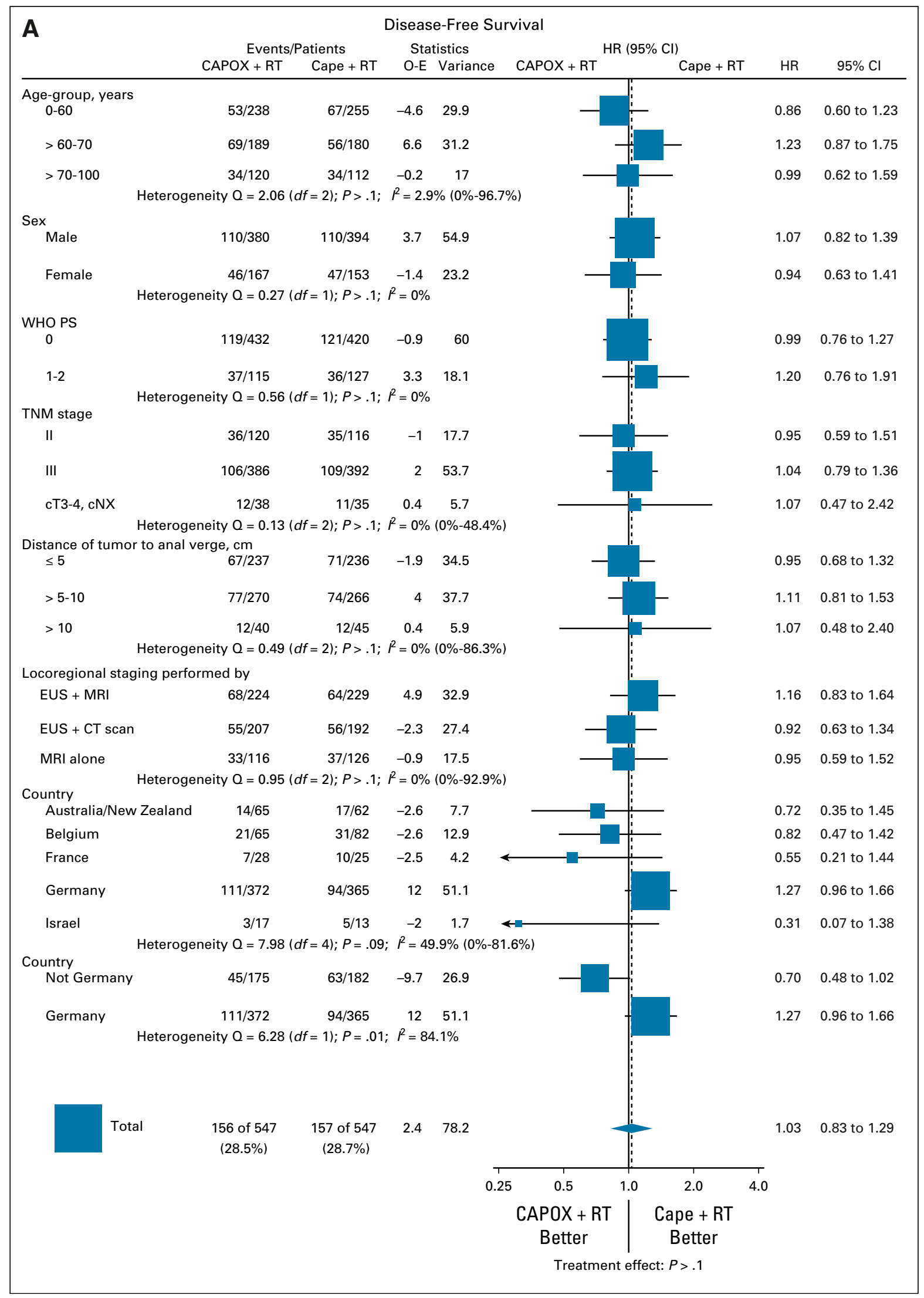

FIG 3. Forest plot of subgroup analyses for disease-free survival and overall survival according to major baseline factors. CT, computed tomography; EUS, endorectal ultrasound; HR, hazard ratio; MRI, magnetic resonance imaging; O-E, XXXX; PS, performance status. 


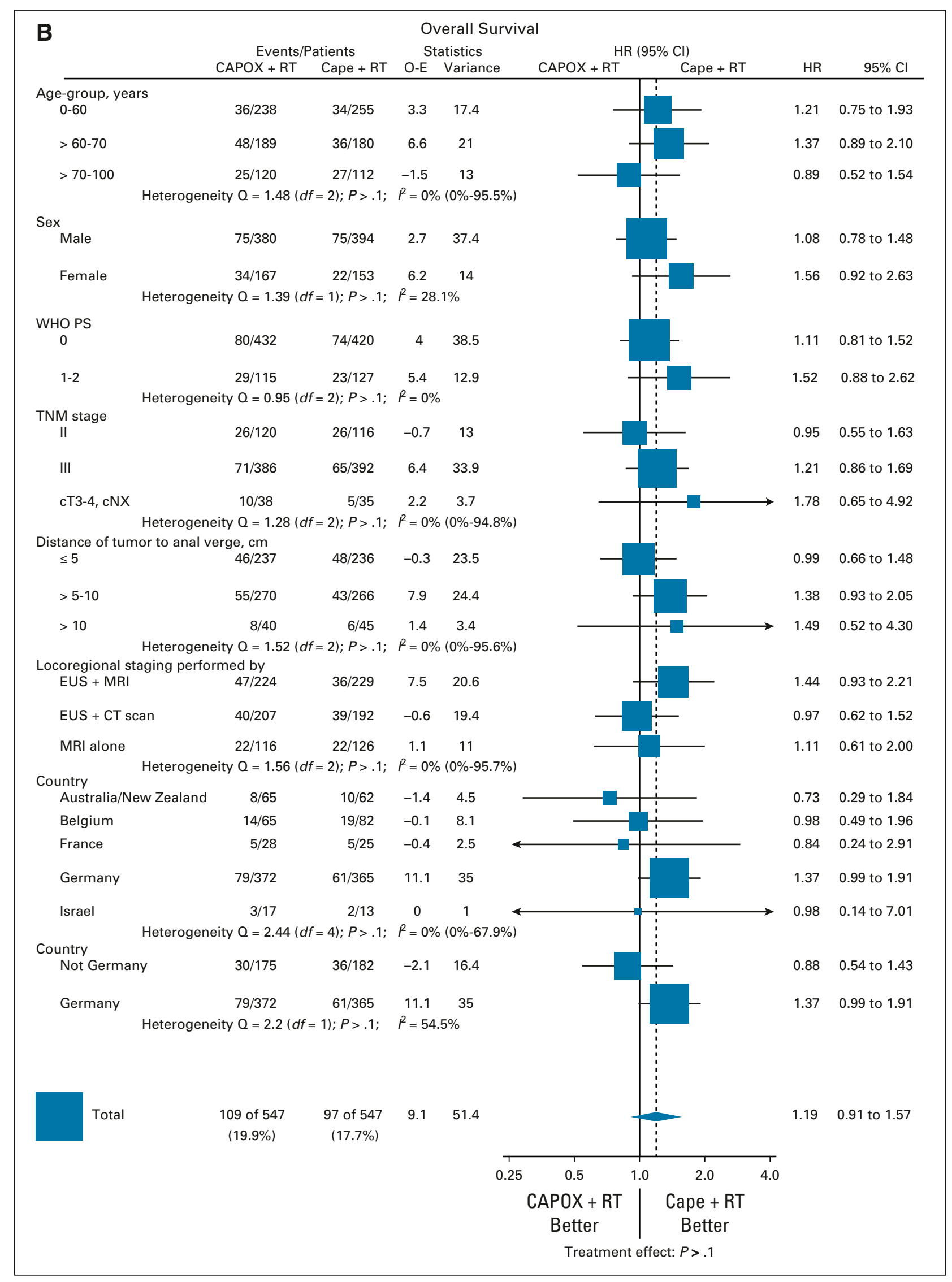

FIG 3. (Continued). 
a low location (no sphincter preservation possible as judged by the surgeon) as independent prognostic factors for a worse DFS (Appendix Table A4, online only). When adding predictive factors (interactions of baseline factors with treatment arm), to be treated outside Germany was the only baseline predictive factor for treatment benefit of oxaliplatin in the multivariable model. In the multivariable analysis, the interaction of country (Germany $v$ not Germany) with treatment remained statistically significant (Appendix Table A5, online only). Therefore, it was not possible to explain the possible heterogeneity in DFS results by these baseline factors.

\section{DISCUSSION}

PETACC 6 was designed to show an increase in DFS by the addition of oxaliplatin to capecitabine-based perioperative treatment. The study could not demonstrate a benefit of adding oxaliplatin to perioperative capecitabine in terms of DFS, OS, local and distant failure, or pathologic and surgical end points. Furthermore, the addition of oxaliplatin resulted in worse tolerability with a higher rate of grade $3 / 4$ toxicities (mainly diarrhea), particularly in the neoadjuvant CRT part with a tripled rate $(6.1 \% v 18.5 \%)$. In contrast to purely adjuvant trials in colon cancer, the rate of grade $3 / 4$ diarrhea remained relevantly higher by the addition of oxaliplatin (5.4\% v12.5\%), likely an effect of the preceding CRT. ${ }^{16}$ Because of the increased toxicity, the capecitabine dose had to be reduced or withdrawn in nearly every third patient in the combination arm compared with $10.5 \%$ in the control arm.

Moreover, the perioperative complication rate was significantly higher in the combination group. Likely, the impaired feasibility of capecitabine in the experimental arm resulted in similar pathologic and surgical secondary end points.

Overall, the PETACC 6 results are in line with other trials that investigated the addition of oxaliplatin to preoperative $\mathrm{CRT}^{4-7,17-19}$ (Appendix Table A6, online only). Four of these five trials showed no or only marginal benefit in terms of $\mathrm{pCR}$ rate or survival end points. Only the CAO/ARO/AIO-04 trial is comparable to PETACC 6 , having had randomly assigned oxaliplatin not only to the neoadjuvant CRT arm but also to the adjuvant treatment arm. In contrast to PETACC 6, a modest, but significant increase in the pCR rate of $4 \%$ and DFS rate $(71.2 \% \vee 75.9 \%$; HR, 0.79 ; $P=.03)$ was shown, with no impact of survival (HR, 0.96). However, compared with PETACC 6, CAO/ARO/AIO-04 applied a different fluoropyrimidine regimen in both arms comparing the CAO/ARO/AIO-94 regimen with continuous infusion 5FU $1,000 \mathrm{mg} / \mathrm{m}^{2}$ on days $1-5$ and $29-33$ (control arm) during RT and four cycles of postoperative bolus $5 \mathrm{FU}$ with $500 \mathrm{mg} / \mathrm{m}^{2}$ on days $1-5$ every 4 weeks with continuous 5FU $250 \mathrm{mg} / \mathrm{m}^{2}$ on days $1-14$ and 22-35 in combination with oxaliplatin $50 \mathrm{mg} / \mathrm{m}^{2}$ on days $1,8,22$, and 29 during RT and postoperatively for eight cycles of a modified infusional 5FU, leucovorin, and oxaliplatin regimen.,
Because there are two variables, the impact of oxaliplatin on the beneficial results is not fully clear. Furthermore, the STAR 1, ACCORD 12/0405-Prodige 2, NSABP R04, and PETACC 6 trials with similar fluoropyrimidine doses and schedules in both arms have not been able to show any difference by the addition of oxaliplatin to preoperative CRT and in PETACC 6, to adjuvant chemotherapy as well. On the basis of the available data comparing capecitabine with $5 F U$ in the perioperative treatment of rectal cancer and capecitabine with bolus 5FU as well as CAPOX with bolus 5FU in the adjuvant setting of UICC stage III colon cancer, capecitabine seems to be more efficacious than $5 \mathrm{FU}$ in the perioperative treatment of colorectal cancer. ${ }^{14,15,20}$ Therefore, it might be speculated that compared with the $\mathrm{CAO} /$ ARO/AIO-04 trial, the PETACC 6 design that applied a highly efficacious control arm with single-agent capecitabine precluded any relevant benefit by the addition of oxaliplatin.

Besides the design issues, the impaired tolerability of the combination arm clearly limited the feasibility of the perioperative treatment. In PETACC 6, only 382 patients (70\% from the baseline population) in the combination arm compared with 421 patients (77\%) in the control arm started adjuvant chemotherapy. Furthermore, the completion rate was lower in the combination arm (76\% v88\%). These different rates, which are in clear contrast to the wellbalanced rates in the CAO/ARO/AIO-04 trial, might also be the reason for the lack of DFS benefit in PETACC 6 . However, oxaliplatin-based adjuvant chemotherapy for postoperatively selected high-risk patients with ypT3/4 or ypN + compared with bolus 5FU and leucovorin resulted in a DFS benefit (HR, 0.63; $P=.018$ ), as currently shown in the randomized phase II ADORE trial. ${ }^{21,22}$

The post hoc subgroup analyses revealed several interesting and not fully explained results. In PETACC 6 , a trend favoring CAPOX in the age-group $\leq 60$ years, although not significant and only marginally, supports the corresponding findings of CAO/ARO/AIO-04, with significant improvement of DFS but without any effect on OS. Of note, the study site location (Germany $v$ not Germany) was predictive for oxaliplatin benefit, with a crossing over of the DFS results in both arms (Data Supplement). Staging, particularly the use of MRI and the application of adjuvant chemotherapy, relevantly differed between German and non-German sites, but this does not explain the strong difference in outcome. In addition, besides the overall favorable clinical T and N stage in Germany, the control group in Germany showed a trend toward favorable clinical and pathologic $\mathrm{T}$ and $\mathrm{N}$ stage compared with the other groups. Although multivariable analysis did not identify potential factors beyond the different outcome for the country of origin, further in-depth analysis is necessary and currently planned. However, careful interpretation of these subgroup analyses is necessary because of the inherent limitations of retrospective analyses. 
In conclusion, perioperative multimodality treatment with CAPOX did not improve pCR, DFS, and OS but resulted in increased toxicity and, therefore, decreased feasibility of preoperative CRT. This trial adds evidence that the addition of oxaliplatin to capecitabine-based CRT adds toxicity without a benefit, which is consistent with most prior experience. Thus, oxaliplatin-based CRT should not be

\section{AFFILIATIONS}

${ }^{1}$ Martin Luther University, Halle, Germany

${ }^{2}$ University Medical Center Hamburg-Eppendorf, Hamburg, Germany

${ }^{3}$ University Hospitals and KU Leuven, Leuven, Belgium

${ }^{4}$ Queen Elizabeth Hospital, Woodville, South Australia, Australia

${ }^{5}$ Universitaetsmedizin Mannheim, Mannheim, Germany

${ }^{6} \mathrm{CHU}$ Ambroise Paré, Assistance Publique-Hôpitaux de Paris, BoulogneBillancourt, France

${ }^{7}$ Université Catholique de Louvain, CHU-UCL-Namur (Sainte-Elisabeth), Namur, Belgium

${ }^{8}$ AZ Turnhout, Turnhout, Belgium

${ }^{9}$ Institute of Oncology, Davidoff Center, Rabin Medical Center, Petah

Tikva, Israel

${ }^{10}$ Sackler Faculty of Medicine, Tel Aviv University, Tel Aviv, Israel

${ }^{11}$ Leopoldina-Krankenhaus der Stadt Schweinfurt gGmbH, Schweinfurt,

Germany

${ }^{12}$ Allgemeines Krankenhaus Celle, Celle, Germany

${ }^{13}$ Klinikum Ludwigsburg, Ludwigsburg, Germany

${ }^{14}$ Onkologische Schwerpunktpraxis, Hanau, Germany

${ }^{15} \mathrm{Gemeinschaftspraxis} \mathrm{Haematologie} \mathrm{und} \mathrm{Onkologie,} \mathrm{Bottrop,} \mathrm{Germany}$

${ }^{16}$ Alfred Health and School of Public Health, Monash University,

Melbourne, Victoria, Australia

${ }^{17}$ Austin-Health, Heidelberg, Victoria, Australia

${ }^{18}$ European Organisation for Research and Treatment of Cancer

Headquarters, Brussels, Belgium

${ }^{19}$ Caritasklinikum, Saarbrucken, Germany

\section{CORRESPONDING AUTHOR}

Hans-Joachim Schmoll, MD, PhD, Clinic for Internal Medicine IV-Hematology/Oncology, University Clinic, Martin Luther University Halle-Wittenberg, Ernst-Grube Str 40, 06120 Halle (Saale), Germany; e-mail: hans-joachim.schmoll@uk-halle.de.

\section{PRIOR PRESENTATION}

Presented at the American Society of Clinical Oncology 2014 Annual Meeting, Chicago, IL, June 3-7, 2014; World Congress on

Gastrointestinal Cancer 2014, Barcelona, Spain, June 25-28, 2014; European Society for Medical Oncology 2016 Congress, Copenhagen, Denmark, October 7-11, 2016; and American Society of Clinical Oncology 2018 Annual Meeting, Chicago, IL, June 1-5, 2018.

\section{SUPPORT}

Supported in Europe by the European Organisation for Research and Treatment of Cancer (EORTC). The study was sponsored in Australia and New Zealand by the Australasian Gastro-Intestinal Trials Group. This trial was conducted by the Arbeitsgemeinschaft Internistische Onkologie; Belgian Group of Digestive Oncology; EORTC Gastrointestinal Tract Cancer Group; EORTC Radiation Oncology Group; Fédération applied. The toxicity of the addition of oxaliplatin to postoperative capecitabine strongly limits the optimal dose, which might have been responsible for the inefficacy of oxaliplatin in this trial. Therefore, if any, the potential of oxaliplatin as part of preoperative chemotherapy is currently being investigated for the development of the total neoadjuvant treatment strategy. ${ }^{23}$

Francophone de Cancérologie Digestive; and the National Health and Medical Research Council Clinical Trials Centre, Australia, and was supported by an educational grant from Hoffmann-La Roche. Additional funding was provided by Pfizer. In Australia, the study was supported by two Cancer Australia priority-driven collaborative cancer research grants (512522 and 1048035) and a Cancer Council New South Wales project grant (RG13-11). The investigational drugs were supplied by Roche and Sanofi-Aventis.

\section{CLINICAL TRIAL INFORMATION NCT00766155 (PETACC-6)}

\section{AUTHORS' DISCLOSURES OF POTENTIAL CONFLICTS OF INTEREST}

Disclosures provided by the authors and data availability statement (if applicable) are available with this article at DOI https://doi.org/10.1200/ JCO.20.01740.

\section{AUTHOR CONTRIBUTIONS}

Conception and design: Hans-Joachim Schmoll, Eric Van Cutsem, Timothy Price, Bernard Nordlinger, John Zalcberg, Karin Haustermans Administrative support: Hans-Joachim Schmoll, Bernard Nordlinger, Stephan Hollerbach, Sandrine Marreaud, Manfred P. Lutz Provision of study material or patients: Hans-Joachim Schmoll, Eric Van Cutsem, Timothy Price, Ralf D. Hofheinz, Baruch Brenner, Karel Caca, Niall Tebbutt, Sandrine Marreaud, Manfred P. Lutz, Karin Haustermans Collection and assembly of data: Hans-Joachim Schmoll, Alexander Stein, Eric Van Cutsem, Ralf D. Hofheinz, Bernard Nordlinger, Jean-François Daisne, Jos Janssens, Baruch Brenner, Hans Reinel, Stephan Hollerbach, Karel Caca, Florian Fauth, Niall Tebbutt, Sandrine Marreaud, Manfred P. Lutz

Data analysis and interpretation: Hans-Joachim Schmoll, Alexander Stein, Eric Van Cutsem, Timothy Price, Ralf D. Hofheinz, John Zalcberg, Niall Tebbutt, Murielle E. Mauer, Sandrine Marreaud, Manfred P. Lutz, Karin Haustermans

Manuscript writing: All authors

Final approval of manuscript: All authors

Accountable for all aspects of the work: All authors

\section{ACKNOWLEDGMENT}

We thank all investigators who participated through the collaborative groups: see list of participating centers in the Appendix. We also thank M.-A. Lentz, data manager of the study at EORTC Headquarters. We thank all patients who participated in this trial, all institutions who included patients (in particular, Peter Schmidt, MD, in Neunkirchen), all the staff engaged in this study, and especially the study team at EORTC and KKS Halle.

\section{REFERENCES}

1. Sauer R, Becker H, Hohenberger W, et al: Preoperative versus postoperative chemoradiotherapy for rectal cancer. N Engl J Med 351:1731-1740, 2004

2. Bosset JF, Collette L, Calais G, et al: Chemotherapy with preoperative radiotherapy in rectal cancer. N Engl J Med 355:1114-1123, 2006 
3. Gérard JP, Conroy T, Bonnetain F, et al: Preoperative radiotherapy with or without concurrent fluorouracil and leucovorin in T3-4 rectal cancers: Results of FFCD 9203. J Clin Oncol 24:4620-4625, 2006

4. Gérard JP, Azria D, Gourgou-Bourgade S, et al: Clinical outcome of the ACCORD 12/0405 PRODIGE 2 randomized trial in rectal cancer. J Clin Oncol 30: 4558-4565, 2012

5. Rödel $\mathrm{C}$, Liersch T, Becker $\mathrm{H}$, et al: Preoperative chemoradiotherapy and postoperative chemotherapy with fluorouracil and oxaliplatin versus fluorouracil alone in locally advanced rectal cancer: Initial results of the German CAO/ARO/AIO-04 randomised phase 3 trial. Lancet Oncol 13:679-687, 2012

6. Allegra CJ, Yothers G, O'Connell MJ, et al: Neoadjuvant 5-FU or capecitabine plus radiation with or without oxaliplatin in rectal cancer patients: A phase III randomized clinical trial. J Natl Cancer Inst 107:djv248, 2015

7. Aschele C, Cionini L, Lonardi S, et al: Primary tumor response to preoperative chemoradiation with or without oxaliplatin in locally advanced rectal cancer: Pathologic results of the STAR-01 randomized phase III trial. J Clin Oncol 29:2773-2780, 2011

8. De Paoli A, Chiara S, Luppi G, et al: Capecitabine in combination with preoperative radiation therapy in locally advanced, resectable, rectal cancer: A multicentric phase II study. Ann Oncol 17:246-251, 2006

9. Dunst J, Reese T, Sutter T, et al: Phase I trial evaluating the concurrent combination of radiotherapy and capecitabine in rectal cancer. J Clin Oncol 20: 3983-3991, 2002

10. Kim JC, Kim TW, Kim JH, et al: Preoperative concurrent radiotherapy with capecitabine before total mesorectal excision in locally advanced rectal cancer. Int J Radiat Oncol Biol Phys 63:346-353, 2005

11. Arkenau HT, Arnold D, Cassidy J, et al: Efficacy of oxaliplatin plus capecitabine or infusional fluorouracil/leucovorin in patients with metastatic colorectal cancer: A pooled analysis of randomized trials. J Clin Oncol 26:5910-5917, 2008

12. Rödel C, Liersch T, Hermann RM, et al: Multicenter phase II trial of chemoradiation with oxaliplatin for rectal cancer. J Clin Oncol 25:110-117, 2007

13. Rödel C, Grabenbauer GG, Papadopoulos T, et al: Phase I/II trial of capecitabine, oxaliplatin, and radiation for rectal cancer. J Clin Oncol 21:3098-3104, 2003

14. Twelves C, Wong A, Nowacki MP, et al: Capecitabine as adjuvant treatment for stage III colon cancer. N Engl J Med 352:2696-2704, 2005

15. Schmoll HJ, Tabernero J, Maroun J, et al: Capecitabine plus oxaliplatin compared with fluorouracil/folinic acid as adjuvant therapy for stage III colon cancer: Final results of the NO16968 randomized controlled phase III trial. J Clin Oncol 33:3733-3740, 2015

16. Haller DG, Tabernero J, Maroun J, et al: Capecitabine plus oxaliplatin compared with fluorouracil and folinic acid as adjuvant therapy for stage III colon cancer. J Clin Oncol 29:1465-1471, 2011

17. O'Connell MJ, Colangelo LH, Beart RW, et al: Capecitabine and oxaliplatin in the preoperative multimodality treatment of rectal cancer: Surgical end points from National Surgical Adjuvant Breast and Bowel Project trial R-04. J Clin Oncol 32:1927-1934, 2014

18. Rödel C, Graeven U, Fietkau R, et al: Oxaliplatin added to fluorouracil-based preoperative chemoradiotherapy and postoperative chemotherapy of locally advanced rectal cancer (the German CAO/ARO/AIO-04 study): Final results of the multicentre, open-label, randomised, phase 3 trial. Lancet Oncol 16: 979-989, 2015

19. Gérard JP, Azria D, Gourgou-Bourgade S, et al: Comparison of two neoadjuvant chemoradiotherapy regimens for locally advanced rectal cancer: Results of the phase III trial ACCORD 12/0405-Prodige 2. J Clin Oncol 28:1638-1644, 2010

20. Hofheinz RD, Wenz F, Post S, et al: Chemoradiotherapy with capecitabine versus fluorouracil for locally advanced rectal cancer: A randomised, multicentre, non-inferiority, phase 3 trial. Lancet Oncol 13:579-588, 2012

21. Hong YS, Nam BH, Kim KP, et al: Oxaliplatin, fluorouracil, and leucovorin versus fluorouracil and leucovorin as adjuvant chemotherapy for locally advanced rectal cancer after preoperative chemoradiotherapy (ADORE): An open-label, multicentre, phase 2, randomised controlled trial. Lancet Oncol 15:1245-1253, 2014

22. Hong YS, Kim SY, Lee JS, et al: Oxaliplatin-Based Adjuvant Chemotherapy for Rectal Cancer After Preoperative Chemoradiotherapy (ADORE): Long-term results of a randomized controlled trial. J Clin Oncol 37:3111-3123, 2019

23. Fokas E, Allgäuer M, Polat B, et al: Randomized phase II trial of chemoradiotherapy plus induction or consolidation chemotherapy as total neoadjuvant therapy for locally advanced rectal cancer: CAO/ARO/AIO-12. J Clin Oncol 37:3212-3222, 2019 
Pre- and Postoperative Capecitabine Without or With Oxaliplatin in Locally Advanced Rectal Cancer: PETACC 6 Trial by EORTC GITCG and ROG, AIO, AGITG, BGDO, and FFCD

The following represents disclosure information provided by authors of this manuscript. All relationships are considered compensated unless otherwise noted. Relationships are self-held unless noted. I = Immediate Family Member, Inst = My Institution. Relationships may not relate to the subject matter of this manuscript. For more information about ASCO's conflict of interest policy, please refer to www.asco.org/rwc or ascopubs.org/jco/authors/author-center.

Open Payments is a public database containing information reported by companies about payments made to US-licensed physicians (Open Payments).

Hans-Joachim Schmoll

Consulting or Advisory Role: Roche, SERVIER, Amgen, Astellas Pharma TREOS, Enterome

Research Funding: Bristol Myers Squibb (Inst), Merck KGaA (Inst), SERVIER (Inst), Roche (Inst), Genentech (Inst), Novartis (Inst)

Travel, Accommodations, Expenses: Roche, Amgen, Bristol Myers Squibb, SERVIER

\section{Alexander Stein}

Consulting or Advisory Role: Merck KGaA, Bristol Myers Squibb, Amgen, Roche, MSD, SERVIER, AstraZeneca

Speakers' Bureau: Roche, Sanofi, Bayer AG, Eli Lilly, Celgene, Amgen, Merck KGaA, SERVIER, Bristol Myers Squibb

Research Funding: Roche (Inst), Sanofi (Inst), Merck KGaA (Inst), Bristol Myers Squibb (Inst), SERVIER (Inst)

Travel, Accommodations, Expenses: Roche, Bristol Myers Squibb, Merck KGaA

Eric Van Cutsem

Consulting or Advisory Role: Bayer AG, Eli Lilly, Roche, SERVIER, Bristol Myers Squibb, Celgene, Merck Sharp \& Dohme, Merck KGaA, Novartis, AstraZeneca, Halozyme, Array BioPharma, Biocartis, GlaxoSmithKline, Daiichi Sankyo, Pierre Fabre, Sirtex, Taiho, Incyte

Research Funding: Amgen (Inst), Bayer AG (Inst), Boehringer Ingelheim (Inst), Eli Lilly (Inst), Novartis (Inst), Roche (Inst), Celgene (Inst), Ipsen (Inst), Merck (Inst), Merck KGaA (Inst), SERVIER (Inst), Bristol Myers Squibb (Inst)

\section{Timothy Price}

Consulting or Advisory Role: Amgen, Roche (Inst), Merck Serono (Inst)

Research Funding: Amgen (Inst)

Travel, Accommodations, Expenses: Amgen

Ralf D. Hofheinz

Honoraria: Roche, Amgen, Merck Serono, Sanofi, MSD, Bristol Myers Squibb, Eli Lilly, Medac, SERVIER, AstraZeneca

Consulting or Advisory Role: Merck Serono, Amgen, Roche, Sanofi, Bristol Myers Squibb, MSD, SERVIER

Speakers' Bureau: Roche, Sanofi, Eli Lilly, Merck, Amgen, SERVIER, MSD, Bristol Myers Squibb

Research Funding: Sanofi (Inst), Medac (Inst), Deutsche Kresbhilfe (Inst)

\section{Baruch Brenner}

Honoraria: MSD Oncology, Bristol Myers Squibb, Roche

Consulting or Advisory Role: MSD Oncology, Boehringer Ingelheim

Research Funding: Merck Serono, Bristol Myers Squibb

Travel, Accommodations, Expenses: Bristol Myers Squibb, Roche, Merck

Serono

Stephan Hollerbach

Research Funding: Interscope (Inst)

Carla V. Hannig

Honoraria: Roche, Takeda Pharmaceuticals, Boehringer Ingelheim, Novartis Travel, Accommodations, Expenses: Celgene, Novartis

\section{John Zalcberg}

Stock and Other Ownership Interests: GW Pharmaceuticals, Aimmune, Vertex, Alnylam, Biomarin, Ophthea, Amarin, Concert Pharmaceuticals, Frequency Therapeutics, Global Blood Therapeutics, Gilead Sciences, Madrigal Pharmaceuticals, UniQure, Sangamo Biosciences, Acceleron Pharmaceuticals, Zogenix, Myovant Sciences, Orphazyme, Moderna Therapeutics

Honoraria: Pfizer, Specialized Therapeutics, Merck Serono, Targovax, Halozyme, Gilead Sciences, Novella Clinical

Consulting or Advisory Role: Pfizer, Merck Serono, Targovax, Merck Sharp \& Dohme, Sirtex Medical, Halozyme, Lipotek, Specialized Therapeutics, CEND Research Funding: Bayer AG (Inst), Merck Serono (Inst), Roche (Inst), Bristol Myers Squibb (Inst), Pfizer (Inst), AstraZeneca (Inst), Specialized Therapeutics (Inst), Baxalta (Inst), Shire (Inst), Eli Lilly (Inst)

Travel, Accommodations, Expenses: Merck Serono, AstraZeneca, Merck Sharp \& Dohme, Deciphera

Niall Tebbutt

Honoraria: Roche, Bristol Myers Squibb

Consulting or Advisory Role: Roche, Bristol Myers Squibb

Research Funding: Bristol Myers Squibb (Inst)

Travel, Accommodations, Expenses: Bayer AG

Manfred P. Lutz

Speakers' Bureau: SERVIER, Eli Lilly

Research Funding: SERVIER (Inst)

Karin Haustermans

Honoraria: Varian Medical Systems

Research Funding: Varian Medical Systems

No other potential conflicts of interest were reported. 


\section{Specifications of Statistical Analysis}

The primary efficacy analyses for this trial were done in all randomly assigned patients according to the intention-to-treat principle. After a median follow-up of 64 months, disease-free survival (DFS) and overall survival (OS) were compared using the Cox proportional hazards regression model adjusted for all stratification factors except center as the primary method of analysis. DFS and OS rates, KaplanMeier method, and treatment effects were summarized with hazard ratios and respective 95\% Cls. The cumulative incidences of locoregional and distant failures were estimated and compared between arms using the competing-risk methodology, with death in the absence of an event treated as a competing risk. Differences between arms were tested using the Fine and Gray model adjusted for all stratification factors but center. For end points pertaining to the patients' surgical outcome, the event rates were compared between arms using a logistic regression model adjusted for all stratification factors but center. Patients not operated on or not receiving a resection were scored as having experienced treatment failure in these analyses.

Possible heterogeneity of the results was investigated by performing subgroup analyses. Heterogeneity was tested by means of a Cochran's $Q$ test. Graphical displays of the results are provided as Forest plots.

A multivariable prognostic analysis was performed on DFS using Cox proportional hazards regression models. The analysis was restricted to the per-protocol population (pooled treatment arms). Interactions of baseline factors with treatment arm were added in the model to allow the identification of possible predictive factors of treatment benefit. For the model building, Collett's model selection approach was used with a level of significance of .1 for the univariable screening and entry and stay criteria of .05 (Collett D: Modelling Survival Data in Medical Research, 1994). The safety analyses were performed on all patients who had started their allocated treatment. All statistical analyses were conducted using SAS 9.4 software (SAS Institute, Cary, NC).

\section{Trial 40054: List of Participating Centers}

Name

\begin{tabular}{|c|c|c|}
\hline Gill, Sanjeev & Alfred Hospital & Australia \\
\hline Tebbutt, Niall & Austin Hospital & Australia \\
\hline Asghari, Ray & Bankstown-Lidcombe Hospital & Australia \\
\hline Briscoe, Karen & Coffs Harbor Health Campus & Australia \\
\hline Karapetis, Chris & Flinders Medical Centre & Australia \\
\hline Begbie, Stephen & Port Macquarie Base Hospital & Australia \\
\hline Goldstein, David & Prince of Wales Hospital & Australia \\
\hline Walpole, Euan & Princess Alexandra Hospital & Australia \\
\hline Price, Timothy & The Queen Elizabeth Hospital & Australia \\
\hline Hruby, George & Royal Prince Alfred Hospital & Australia \\
\hline Ng, Siobhan & St John of God Hospital, Subiaco & Australia \\
\hline Lynch, Rod & Geelong Hospital-Andrew Love Cancer Centre & Australia \\
\hline Sabesan, Sabe & Townsville General Hospital & Australia \\
\hline Monsaert, Els & AZ Maria Middelares & Belgium \\
\hline Vanderstraeten, Erik & AZ Maria Middelares & Belgium \\
\hline Ferrante, Michel & AZ Sint-Maarten & Belgium \\
\hline Janssens, Jozef & AZ Turnhout-Campus Sint Elisabeth & Belgium \\
\hline Martens, Michel & AZ Turnhout-Campus Sint Elisabeth & Belgium \\
\hline
\end{tabular}

(continued on following page) 
Daisne, Jean-François

Weytjens, Reinhilde

Delaunoit, Thierry

Peeters, Marc

Van Den Brande, Jan

Laurent, Stéphanie

Van Den Weyngaert, Danielle

Vanderkam, Sabine

Schrijvers, Dirk

Martin, Philippe

Crehange, Gilles

Truc, Gilles

Karine, Peignaux

Maingon, Philippe

Artignan, Xavier

Desseigne, Françoise

Martel-Lafay, Isabelle

Latrive, Jean-Paul

Bosset, Jean-François

Nguyen, Thierry

Etienne, Pierre

Ducreux, Michel

Seitz, Jean-François

Ben Abdelghani, Meher

Freier, Werner

Hollerbach, Stephan

Peters, Uwe

Holtkamp, Wilhelm

Speidel, Andrea

Thiemann, Roland

Behringer, Dirk

Gaska, Tobias

Kremers, Stephan

Matzdorf, Axel

Moser, Lutz

Mueller-Naendrup, Clemens

Pflueger, Karl-Heinz

Reymond, Marc Andre

Potenberg, Jochem

Baake, Gerold

Esser, Martin

Held, Harald

Soeling, Ulrike
CHU Site Sainte-Elisabeth-UCL Namur

Belgium

GasthuisZusters Antwerpen-Sint-Augustinus

Belgium

Hopital De Jolimont

Universitair Ziekenhuis Antwerpen

Universitair Ziekenhuis Antwerpen

Universitair Ziekenhuis Gent

ZNA Middelheim

ZNA Middelheim

ZNA Middelheim

Centre Bourgogne

Centre Georges-François-Leclerc

Centre Georges-François-Leclerc

Centre Georges-François-Leclerc

Belgium

Belgium

Belgium

Belgium

Belgium

Belgium

Belgium

France

France

France

France

Centre Georges-François-Leclerc

France

Centre Hospitalier Prive Saint-Gregoire

France

Centre Leon Berard

Centre Leon Berard

France

France

$\mathrm{CH}$ de Compiegne

France

CHRU de Besancon-Hopital Jean Minjoz

France

CHRU de Besancon-Hopital Jean Minjoz

France

Clinique Armoricaine De Radiologie

France

Gustave Roussy

France

Hôpital de La Timone (APHM)

France

Institut de Cancerologie Strasbourg Europe (formerly Paul Strauss)

France

Onkologie im Medicinum

France

Allgemeines Krankenhaus Celle

Germany

Ambulantes Tumorzentrum Spandau

Germany

Ammerland-Klinik GmbH-Universitaet Goettingen

Germany

Arztefor. Hennigsdor

Germany

Asklepios Klinik Weissenfels

Germany

Augusta-Kranken-Anstalt

Germany

Bruederkrankenhaus St Josef Paderborn

Germany

Caritas KRHS Lebach

Germany

CaritasKlinikum Saarbruecken St Theresia

Charite-Universitaetsmedizin Berlin-Campus Benjamin Franklin

Germany

Germany

Darmzentrum Suedwestfalen, Katholische Hospitalgesellschaft

Germany

Ev Diakonie-Krankenhaus gGmbH

Germany

Ev Johannes-Krankenhaus

Ev Waldkrankenhaus Spandau

Germany

Facharzt fuer Innere Medizin

Germany

Facharzt fuer Innere Medizin

Germany

Germany

Friedrich-Ebert-Krankenhaus

Germany

Gemeinschaftspraxis

Germany

(continued on following page) 
(continued)

Dingeldein, Gerrit

Bangerter, Markus

Jacobasch, Lutz

Hannig, Carla Verena

Reiser, Marcel

Schmidt, Burkhard

Tschechne, Barbara

Bertram, Mathias

Brinkmann, Lutz

Forstbauer, Helmut

Engel, Erik

Hegewisch-Becker, Susanna

Van Roye, V.

Boicev, Alexander

Alberti, Winfried

Halm, Ulrich

Boldt, Thomas

Reeb, Manfred

Niedermeier, Michael

Hoesl, Mark

Frank-Gleich, Stefanie

Ko, Yon

Hennemann, Burkhard

Doerner, Arnulf

Fischbach, Cathie

Huegle, Ulrich

Schlichthaerle, Tessa

Stahl, Michael Konrad

Kuckhoff, Michael

Hielscher, Joerg

Wagner, Siegfried

Brugger, Wolfram

Baeumer, Sabine

Lambertz, Helmut

Burk, Martin

Gruenewald, Martin

Roemmele, U.

Bildat, Stephan

Kempf, Barbara

Constantin, Christian

Caca, Karel

Wilhelm, Martin

Hebart, Holger
Gemeinschaftspraxis-Darmstadt

Gemeinschaftspraxis Augsburg

Germany

Germany

Gemeinschaftspraxis Dresden

Gemeinschaftspraxis Haematologie und Onkologie

Germany

Gemeinschaftspraxis Katay/Reiser

Germany

Germany

Gemeinschaftspraxis Muenchen

Germany

Gemeinschaftspraxis Tschechne/Luft für Innere Medizin

Germany

Gemeinschaftspraxis-Hamburg

Germany

Gemeinschaftspraxis-Laatzen

Gesellschaft für onkologische Studien, Praxismanagement und Logistik

Germany

Haematologisch-Onkologische Praxis Altona

Germany

Haematologisch-Onkologische Praxis Eppendorf

Germany

Haematologisch-Onkologische Praxis-Koblenz

Germany

Heinrich-Braun-Krankenhaus

Germany

Germany

Helios Klinikum Wuppertal

Germany

Helios Park-Klinikum Leipzig

Germany

Hospital Dresden-Friedrichstadt

Institut für Medizinische Dokumentation, Gutachtenerstellung,

Gesundheitsfoerderung und Qualitaetssicherung GbR

Internistische Gemeinschaftspraxis- Memmingen

Internistische Gemeinschaftspraxis-Nuernberg

Internistisch-Onkologische Gemeinschaftspraxis

Germany

Germany

Johanniter-Krankenhaus Bonn

Johanniter-Krankenhaus Rheinhausen

KKH Alten Eichen

Klinikum Aschaffenburg

Kliniken der Stadt Koeln-Krankenhaus Holweide

Germany

Germany

Germany

Germany

Germany

Germany

Germany

Kliniken der Stadt Koeln-Krankenhaus Holweide

Germany

Kliniken Essen-Mitte

Germany

Klinikum Barnim GmbH

Germany

Klinikum Chemnitz gGmbH

Germany

Germany

Klinikum Deggendorf

Germany

Klinikum Der Stadt Villingen-Schwenningen

Germany

Klinikum Dortmund gGmbH

Klinikum Garmisch-Partenkirchen

Germany

Germany

Klinikum Hanau

Klinikum Heidenheim

Germany

Germany

Klinikum Kirchheim-Nuertingen

Germany

Klinikum Kreis Herford

Germany

Klinikum Landshut

Klinikum Lippe Lemgo

Germany

Klinikum Ludwigsburg

Germany

Klinikum Nuernberg-Standort Nord

Germany

Klinikum Schwaebisch-Gmuend

Germany

Germany

(continued on following page) 


\begin{tabular}{|c|c|c|}
\hline Leser, Hans-Georg & Klinikum Sindelfingen-Boeblingen & Germany \\
\hline Fischer von Weikersthal, Ludwig & Klinikum St Marien & Germany \\
\hline Mergenthaler, Hans-Guenther & Klinikum Stuttgart-Katharinenhospital & Germany \\
\hline Trenn, Guido & Knappschaftskrankenhaus Bottrop & Germany \\
\hline Krummenerl, Patrick & Krankenhaus Martha-Maria Halle Doelau gGmbH & Germany \\
\hline Kirchner, Hartmut; Wildfang, I. & Krankenhaus Siloah-Klinikum Region Hannover GmbH & Germany \\
\hline Gosenheimer, Robert & Krankenhaus St Marienwoerth & Germany \\
\hline Grunewald, Martina & Kreisklinik Aschersleben-Stassfurt gGmbH & Germany \\
\hline Sieber, Markus & Kreiskrankenhaus Gummersbach GmbH & Germany \\
\hline Reinel, Hans & Leopoldina-Krankenhaus der Stadt Schweinfurt gGmbH & Germany \\
\hline Hapke, Gunnar & Marienkrankenhaus & Germany \\
\hline Arnold, Dirk & Martin Luther Universitaet-Universitaetsklinikum Halle (Saale) & Germany \\
\hline Schmoll, Hans-Joachim & Martin Luther Universitaet-Universitaetsklinikum Halle (Saale) & Germany \\
\hline Koenigsmann, Michael & Mediprojekt GbR-Hannover & Germany \\
\hline Clemens, Michael & Mutterhaus Der Borromaerinnen & Germany \\
\hline Mahlberg, Rolf & Mutterhaus Der Borromaerinnen & Germany \\
\hline Demandt, Matthias & MVZ Klinikum Straubing GmbH & Germany \\
\hline Grossmann, Johannes & Notdienstpraxis am Evangelischen Krankenhaus Bethesda & Germany \\
\hline Eggert, Jochen & OnkoLog Moers GbR & Germany \\
\hline Rauh, Jacqueline & Onkologische Schwerpunktpraxis Dr Koch & Germany \\
\hline Ladda, Ekkehart & Onkologische Schwerpunktpraxis Neumarkt & Germany \\
\hline Fauth, Florian & Onkologische Schwerpunktpraxis-Hanau & Germany \\
\hline Goehler, Thomas & Onkozentrum Dresden/Freiberg & Germany \\
\hline Woerdehoff, Herbert & Otto-Von-Guericke-Universitaet Magdeburg-Universitaetsklinik & Germany \\
\hline Geiger, Matthias & Paracelsius Krankenhaus & Germany \\
\hline Breuer, Friedhelm & $\mathrm{PIOH}$ Gemeinschaftspraxis & Germany \\
\hline Peveling Reddemann, Christina & Praxis-Leverkusen & Germany \\
\hline Marquard, Felix & Praxis Dr Marquard & Germany \\
\hline Schroeder, Detlev & Praxis Dr Schroeder & Germany \\
\hline Schmitz, Stephan & Praxis Drs Schmitz/Steinmetz & Germany \\
\hline Dietze, Lutz & Praxis fuer Haematologie und Internistische Onkologie & Germany \\
\hline Schliesser, Georg Christian & Praxis fuer Haematologie und Internistische Onkologie & Germany \\
\hline Papke, Jens & Praxis Innere Medizin-Neustadt & Germany \\
\hline \multicolumn{3}{|c|}{ (continued on following page) } \\
\hline
\end{tabular}


(continued)

\begin{tabular}{|c|c|c|}
\hline Schwerdtfeger, Michael & Praxis Koethen & Germany \\
\hline Groschek, Mathias & Praxis Wuerselen & Germany \\
\hline Hoehler, Thomas & Prosper-Hospital Recklinghausen & Germany \\
\hline Puchtler, Gerhard & RoMed Klinikum Rosenheim & Germany \\
\hline Schmoecker, Christoph & Sana Klinikum Berlin Lichtenberg & Germany \\
\hline Seipelt, Gernot & Schwerpunktpraxis und Tagesklinik & Germany \\
\hline Martens, Uwe & SLK-Kliniken Heilbronn & Germany \\
\hline Schlegel, Frank & St Antonius Hospital & Germany \\
\hline Kaiser, Ulrich & St Bernward Krankenhaus Hildesheim & Germany \\
\hline Staiger, Hans-Juergen & Stadtklinik Baden-Baden & Germany \\
\hline Guenther, Barbara & Staedtisches Kliniken Duesseldorf-Benrath & Germany \\
\hline Schmidt, Peter & Staedtisches Klinikum Neunkirchen & Germany \\
\hline Grothe, Wilfried & Staedtisches Klinikum & Germany \\
\hline Hoeffkes, Heinz-Gert & Staedtisches Klinikum & Germany \\
\hline Deckert, Markus & Staedtisches Klinikum Brandenburg GmbH & Germany \\
\hline Guenter, Andreas & Staedtisches Klinikum Braunschweig gGmbH & Germany \\
\hline Florschuetz, Axel & Staedtisches Klinikum Dessau & Germany \\
\hline Naumann, Ralph & Stiftungsklinikum Mittelrhein & Germany \\
\hline Block, Andreas & Universitaets-Krankenhaus Eppendorf & Germany \\
\hline Hofheinz, Ralf Dieter & UniversitaetsMedizin Mannheim & Germany \\
\hline de Wit, Maike & Vivantes Klinikum Neukoelln & Germany \\
\hline Spaeth-Schwalbe, Ernst & Vivantes Klinikum Spandau & Germany \\
\hline Link, Hartmut & Westpfalz-Klinikum Gmbh & Germany \\
\hline Fronhoffs, Stefan & Zentrum fuer ambulante Haematologie \& Onkologie & Germany \\
\hline Brenner, Baruch & Rabin Medical Center-Tel Aviv & Israel \\
\hline Ben-Yosef, Rahamim & Rambam Health Care Campus, Oncology Institute & Israel \\
\hline Shulman, Katerina & Rambam Health Care Campus, Oncology Institute & Israel \\
\hline Jeffery, Mark & Christchurch Hospital & New Zeal \\
\hline
\end{tabular}


TABLE A1. Correlation Between Dose Reduction or Withdrawal of Capecitabine and/or Oxaliplatin Because of Toxicity and Surgical Outcome

Dose Reduction or

\section{Withdrawal for Toxicity,}

No. (\%)

\begin{tabular}{llll}
\cline { 2 - 3 } Variable & \multicolumn{1}{c}{ No } & \multicolumn{1}{c}{ Yes } & Total, No. (\%) \\
\hline No. of patients & 345 & 202 & 547 \\
\hline Total mesorectal excision & $281(81.4)$ & $281(81.4)$ & $281(81.4)$ \\
\hline R0 (complete) & $320(92.8)$ & $320(92.8)$ & $320(92.8)$ \\
\hline Pathologic complete remission & $45(13.0)$ & $45(13.0)$ & $45(13.0)$
\end{tabular}


TABLE A2. Seven-Year DFS and OS According to Application Versus No Application of Adjuvant Chemotherapy by German and Non-German Patient Population

Cape + RT, \%

CAPOX + RT, \%

Application of Adjuvant Chemotherapy

No. of Patients

Patient Rate Rate at 7 Years

Patient Rate Rate at 7 Years

HR $(95 \% \mathrm{Cl})$

DFS

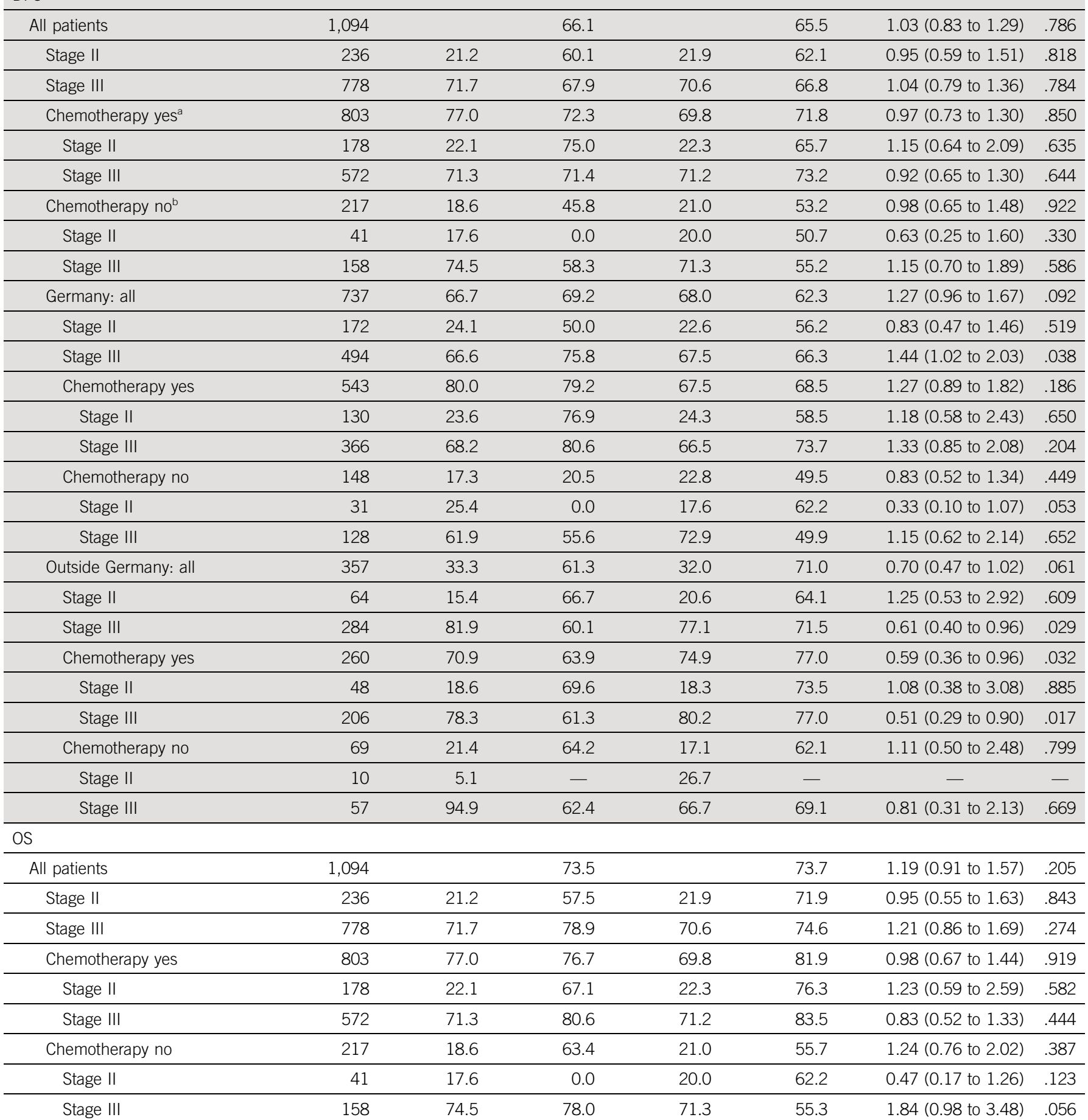

Abbreviations: Cape, capecitabine; CAPOX, capecitabine and oxaliplatin; DFS, disease-free survival; HR, hazard ratio; OS, overall survival; RT, radiotherapy.

aPatients operated within protocol who started adjuvant chemotherapy.

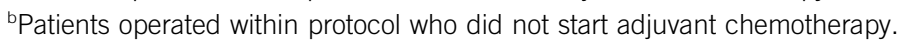


TABLE A3. Baseline and Treatment Characteristics of Patients Enrolled at German and Non-German Sites

Country, No. (\%)

\begin{tabular}{|c|c|c|}
\hline & & \\
\hline Characteristic & Outside Germany & Germany \\
\hline No. of patients & 357 & 737 \\
\hline Median age, years (range) & $61(26-87)$ & $62(23-84)$ \\
\hline Sex & & \\
\hline Male & $248(69.5)$ & $526(71.4)$ \\
\hline Female & 109 (30.5) & 211 (28.6) \\
\hline ECOG PS & & \\
\hline 0 & $282(79.0)$ & $570(77.3)$ \\
\hline 1 & $74(20.7)$ & $160(21.7)$ \\
\hline 2 & $1(0.3)$ & $7(0.9)$ \\
\hline cT & & \\
\hline cT1 & $0(0.0)$ & $5(0.7)$ \\
\hline сT2 & $21(5.9)$ & $48(6.5)$ \\
\hline сT3 & $305(85.4)$ & $630(85.5)$ \\
\hline cT4 & $31(8.7)$ & $54(7.3)$ \\
\hline $\mathrm{cN}$ & & \\
\hline cNO & $64(17.9)$ & $174(23.6)$ \\
\hline $\mathrm{cN1}$ & $195(54.6)$ & $396(53.7)$ \\
\hline $\mathrm{cN} 2$ & $90(25.2)$ & $101(13.7)$ \\
\hline cNX & $8(2.2)$ & $66(9.0)$ \\
\hline TNM stage & & \\
\hline II & $64(17.9)$ & $172(23.3)$ \\
\hline III & $284(79.6)$ & $494(67.0)$ \\
\hline cT3-4, cNX & $8(2.2)$ & $65(8.8)$ \\
\hline Missing & $1(0.3)$ & $6(0.8)$ \\
\hline Distance of tumor to anal ve & & \\
\hline$\leq 5$ & $167(46.8)$ & $306(41.5)$ \\
\hline$>5$ & $190(53.2)$ & $431(58.5)$ \\
\hline MRI available at the center & & \\
\hline No & $36(10.1)$ & $86(11.7)$ \\
\hline Yes & $321(89.9)$ & 651 (88.3) \\
\hline Locoregional staging perforr & & \\
\hline $\mathrm{EUS}+\mathrm{MRI}$ & $129(36.1)$ & $324(44.0)$ \\
\hline EUS + CT scan & $67(18.8)$ & $332(45.0)$ \\
\hline MRI alone & $161(45.1)$ & $81(11.0)$ \\
\hline Adjuvant chemotherapy star & & \\
\hline Cape + RT & $129(70.9)$ & $292(80.0)$ \\
\hline CAPOX + RT & $132(75.4)$ & $251(67.5)$ \\
\hline
\end{tabular}

Abbreviations: Cape, capecitabine; CAPOX, capecitabine and oxaliplatin; CT, computed tomography; EUS, endorectal ultrasound; MRI, magnetic resonance imaging; RT, radiotherapy. 
TABLE A4. Multivariable Prognostic Model for DFS in the Per-Protocol Population Baseline Factor

\begin{tabular}{|c|c|c|}
\hline \multicolumn{3}{|l|}{ Treatment arm } \\
\hline Cape + RT & 1.00 & $.914(1)$ \\
\hline CAPOX + RT & 0.99 (0.78 to 1.24$)$ & \\
\hline \multicolumn{3}{|l|}{ Age, years } \\
\hline$\leq 60$ & 1.00 & $.030(1)$ \\
\hline$>60$ & 1.30 (1.03 to 1.64$)$ & \\
\hline \multicolumn{3}{|l|}{ cT } \\
\hline cT1-cT2 & 1.00 & $.044(2)$ \\
\hline cT3 & 1.67 (0.96 to 2.92) & .071 \\
\hline cT4 & 2.31 (1.19 to 4.48$)$ & .013 \\
\hline \multicolumn{3}{|c|}{ Sphincter preservation according to the surgeon } \\
\hline Not sphincter preserving & 1.00 & $.032(1)$ \\
\hline Sphincter preserving & 0.76 (0.59 to 0.98$)$ & \\
\hline
\end{tabular}

NOTE. The per-protocol population included all eligible patients who started their allocated treatment.

Abbreviations: Cape, capecitabine; CAPOX, capecitabine and oxaliplatin; DFS, disease-free survival; HR, hazard ratio; RT, radiotherapy.

TABLE A5. Treatment Benefit by Predictive Factor Level Adjusted for Identified Prognostic Factors

Treatment Benefit for Perioperative Oxaliplatin ${ }^{\mathrm{a}}$

Test for Predictive Value (interaction) ${ }^{\mathrm{a}}$

\begin{tabular}{|c|c|c|c|}
\hline \multirow[b]{2}{*}{ Country } & & \multirow[b]{2}{*}{$P$} \\
\hline & HR $(95 \% \mathrm{Cl})^{\mathrm{b}}$ & $P$ & \\
\hline Germany & 1.19 (0.90 to 1.59$)$ & .2243 & .0289 \\
\hline Not Germany & 0.69 (0.47 to 1.03$)$ & .0692 & \\
\hline
\end{tabular}

Abbreviation: HR, hazard ratio.

${ }^{a}$ Treatment benefit computed from the multivariable model with prognostic factors age, $\mathrm{cT}$, sphincter preservation according to the surgeon, country (Germany $v$ not Germany), treatment arm, and the interaction of country (Germany $v$ not Germany) and treatment arm.

${ }^{\mathrm{b}}$ Capecitabine and oxaliplatin plus radiotherapy/capecitabine plus radiotherapy. 


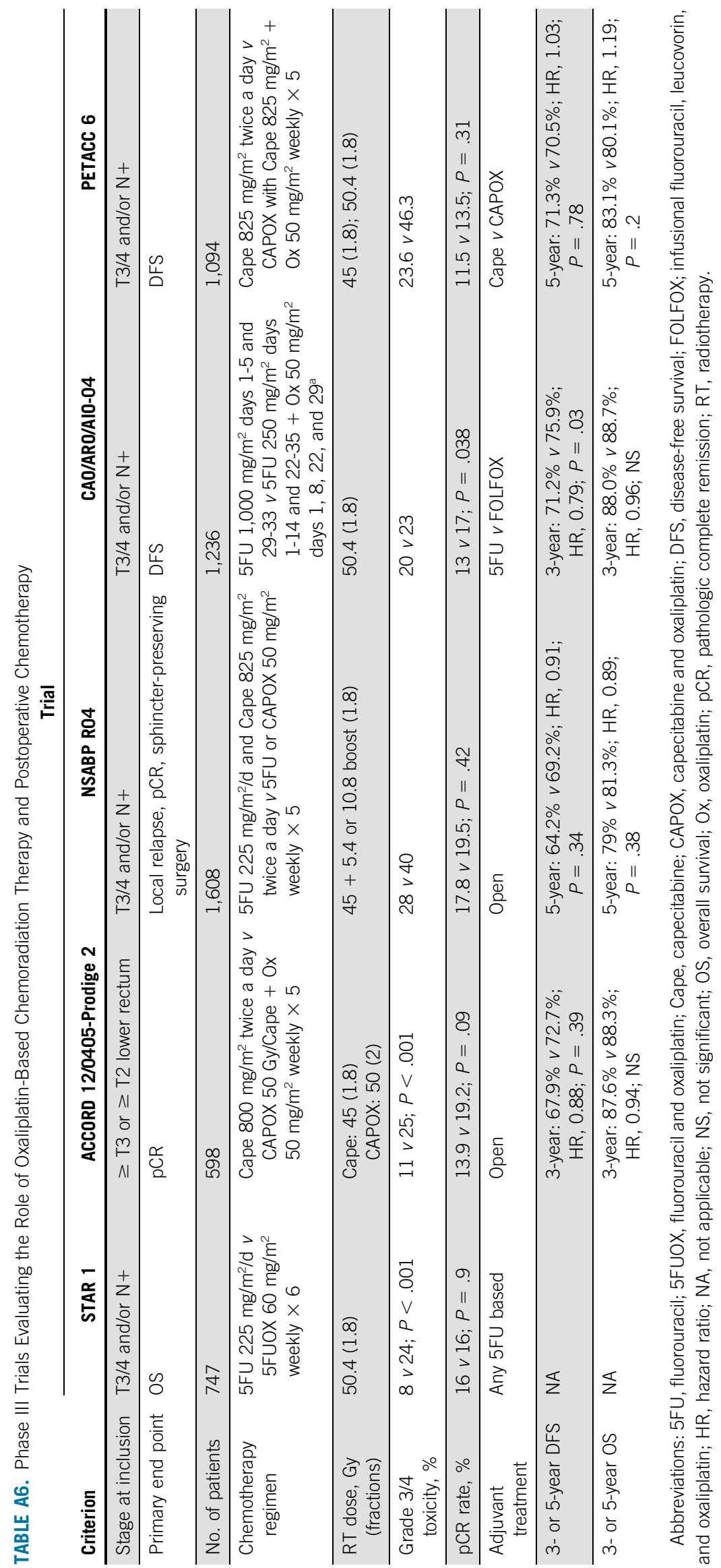

